\title{
The new era of minimally invasive interventions for prostate cancer: robot-assisted laparoscopic radical prostatectomy and focal therapy
}

This article was published in the following Dove Press journal:

Open Access Surgery

6 October 2011

Number of times this article has been viewed

\author{
Oscar Schatloff' \\ Alyssa S Louis ${ }^{2}$ \\ Uri Lindner ${ }^{2}$ \\ 'Global Robotics Institute, Florida \\ Hospital Celebration Health, \\ Celebration, FL, USA; ${ }^{2}$ Division of \\ Urology, Department of Surgical \\ Oncology, University Health Network, \\ Toronto, ON, Canada
}

\begin{abstract}
Prostate cancer remains a common but highly treatable disease. Innovations in prostate cancer treatment have allowed a transition toward minimally invasive approaches in an attempt to avoid treatment-related morbidities. In the middle of the treatment spectrum between radical open surgeries and active surveillance lies robot-assisted laparoscopic radical prostatectomy (RALP) and focal therapy (FT). This review sets out the most current information on RALP and FT (including laser ablation, cryotherapy, high-intensity focused ultrasound, photodynamic therapy, and irreversible electroporation).
\end{abstract}

Keywords: PCa, RALP, FT, laser ablation, cryotherapy, high-intensity focused ultrasound, photodynamic therapy, irreversible electroporation

\section{Introduction}

Prostate cancer (PCa) is the most common nondermatological malignancy in males, with an estimated annual incidence of approximately 220,000 cases in the United States and 300,000 in Europe. ${ }^{1,2}$ Data from the SEER (Surveillance, Epidemiology, and End Results) registry indicate that the incidence of PCa in men under 50 years has risen steadily over the past 10 years, with an annual incidence of $9.5 \%,{ }^{3}$ suggesting that more men will live a long time with the outcomes of their treatment. ${ }^{3,4}$ The goals of active therapy are oncologic control with minimal morbidities, and the latter has driven research on newer, minimally invasive approaches to $\mathrm{PCa}$ treatment. In the paradigm of radical therapy, radical prostatectomy remains the gold standard for localized disease, and several minimally invasive modifications have been added to the original technique including the robot-assisted laparoscopic approach. ${ }^{5}$ The newly emerging paradigm of focal therapy (FT) can be viewed as the middle ground between radical therapy and active surveillance (AS), offering the possibility of less morbidity than any other contemporary technique, while attempting to attain adequate oncologic control. Minimally invasive robot-assisted laparoscopic prostatectomy (RALP) and the rationale and practical considerations of FT will be discussed.

\section{Materials and methods}

The authors of this review performed a PubMed search for articles in English, using the terms robot assisted radical prostatectomy and focal therapy as an unrestricted search. Relevant articles regarding novelties in these two fields were reviewed and are presented below. 


\section{RALP}

Since the first case performed by Binder and Kramer in $2001,{ }^{6}$ RALP has dramatically changed the surgical management of PCa. RALP is an established treatment modality for $\mathrm{PCa}$, accounting for approximately $85 \%$ of radical prostatectomies currently performed in the United States. With the advantages of three-dimensional (3D) vision, tremor filtering, seven degrees of freedom, coordinated eye-hand movement, and superior surgeon ergonometrics when compared with standard laparoscopy, robotic technology offers the potential of performing surgery in a precise and delicate way, especially in places with difficult access such as the male pelvis. However, the supposition that technological breakthroughs will be translated into improved outcomes has to undergo rigorous inspection and validation. In this context, the literature regarding RALP is becoming increasingly mature. With increased surgical experience, well designed prospective studies comparing RALP with open radical prostatectomy (ORP) have been recently published. In addition, a topic in the robotic literature which has received increasing attention is the way to report the results of radical prostatectomy. Patients undergoing surgery nowadays are at the prime of their life, and expect complete cancer cure and functional recovery. In this context, patients expect to be counseled regarding all three goals of cancer control, urinary incontinence, and erectile dysfunction. Below is a review of the most recently reported outcomes and concepts related to RALP.

\section{Current outcomes of RALP}

Although results of high volume centers have demonstrated that RALP offers results at least equivalent to those of ORP, ${ }^{7}$ the reproducibility of these findings in centers with lower caseload has been a matter of long debate. The publication of high impact studies, which have generally used poor methodology, have further increased doubts about the real benefit of robotic prostatectomy. ${ }^{8}$ Even though randomized trials provide the strongest evidence when comparing different treatment modalities, nowadays patient preference regarding treatment options is influenced by many factors, and there is real doubt whether such a study can be performed anymore. In the absence of randomized trials comparing ORP and RALP, the strongest evidence comes from prospective comparative nonrandomized trials. ${ }^{7}$ In this context, two well designed studies from centers with average caseload have been recently published.

A prospective nonrandomized trial by Ficarra et $\mathrm{al}^{9}$ compared 105 patients who underwent ORP with 103 who had RALP. Patients in both arms were under the same data collection protocol, including serial validated questionnaires to assess urinary continence (ICIQ-UI), potency (IIEF-5) and complications (Clavien). Urinary and sexual functions were evaluated before surgery and at 12 months. Patients in the robotic arm were younger (61 versus 65 years; $P<0.01$ ); the remaining preoperative characteristics including performance status, body mass index (BMI), prostate-specific antigen (PSA), and tumor stage and grade did not differ between the study arms. Main results for RALP versus ORP were: overall positive surgical margins (PSMs), $34 \%$ versus $21 \%$ ( $P=$ not significant $[\mathrm{NS}]$ ); PSM in $\mathrm{pT} 2$ disease, $12 \%$ versus $12 \%(P=\mathrm{NS})$; perioperative blood transfusions, $1.9 \%$ versus $14.0 \%(P<0.01)$; perioperative complications, $10 \%$ versus $11 \%(P=\mathrm{NS})$; continent at catheter removal, $69 \%$ versus $41 \%(P<0.001)$; continent at 12 months, $97 \%$ versus $88 \%(P<0.01)$; and mean time to continence, 25 versus 75 days $(P<0.001)$. Among preoperative potent patients who underwent bilateral nerve sparing, $81 \%$ and $49 \%$ of those who underwent RALP and ORP were potent at 12 months. Similarly, the mean time to recovery of erectile function was 3.9 months versus 6.7 months respectively. An interesting aspect of this study is that surgeons from both study arms had different levels of experience. For example, the two robotic surgeons were during their learning curve, with a prior experience of 50 cases each, while the open surgeons had a prior experience of 400 cases each. While this certainly can explain some particular aspects of the results, such as the higher rate of PSM in the robotic group, improved functional outcomes seen with even relatively limited experience are encouraging.

In a study by Di Pierro et al, ${ }^{10}$ the authors prospectively included 150 consecutive radical prostatectomies using the same inclusion and outcome measurements. The first 75 were done in an open fashion by three different surgeons with a prior experience of 100 cases each, while the second 75 were done robotically by a single surgeon with comprehensive open experience and after a 6-month fellowship in robotic and laparoscopic surgery. Preoperative characteristics including age, PSA, and D'Amico risk group were comparable between the study groups. Patients in the robotic arm had longer operative time (330 minutes versus 253 minutes, $P<0.02$ ), less node count after lymphadenectomy (12 versus $18, P<0.001$ ), lower PSM (16\% versus $32 \%, P<0.001$ ), improved 3- and 12-month continence rates $(95 \%$ versus $83 \%, P=0.003$; and $89 \%$ versus $80 \%, P=0.09$ ), and improved 3- and 12-month recovery of erectile function (68\% versus $25 \%, P=0.001$; and $55 \%$ versus $26 \%, P=0.009$ ). Although overall complication rates were similar (RALP 
$40 \%$ versus ORP $37 \%$ ), those occurring in the robotic group were significantly less severe, with $84 \%$ being Clavien I or II, versus $46 \%$ in the open group, $P=0.005$ ).

\section{New concepts in reporting results of radical prostatectomy}

The goal of radical prostatectomy has been traditionally described as the ability to excise the cancer completely while achieving good recovery of urinary control and erectile function. While each of the former outcomes has been extensively reported individually, the concept of reporting the likelihood of achieving these three outcomes concurrently, the so called "trifecta," has gradually spread since first reported by Bianco et al. ${ }^{11}$ Subsequently, Eastman et al ${ }^{12}$ evaluated the trifecta outcomes in 1577 patients who underwent ORP. The trifecta was achieved in $62 \%$ of patients at 48 months of follow-up. Patel et al ${ }^{13}$ reported the trifecta outcome in 404 patients who underwent RALP. The trifecta was achieved in $80.3 \%$ of patients at 12 months.

As $\mathrm{PCa}$ is more frequently diagnosed in younger and healthier men, the current demands and expectations of patients who desire surgical treatment of $\mathrm{PCa}$ are much higher and cannot be addressed with the current trifecta outcomes. For example, a patient with a rectal injury and temporary colostomy after RALP can still reach the trifecta. Therefore, patients who have reached the trifecta but have also experienced postoperative complications or positive surgical margins might not be completely satisfied after surgery. In this context, Patel et a ${ }^{14}$ proposed a new concept for reporting outcomes after radical prostatectomy: the "pentafecta," which includes the outcomes of the trifecta in addition to negative surgical margins and absence of perioperative complications. In their report on 332 patients, the pentafecta was reached in $70.8 \%$ of the patients by 12 months.

The outcomes of RALP are encouraging when compared with traditional open methods, but more studies with adequate methodology and larger cohorts are warranted. This will allow more definitive conclusions about the advantages of RALP and its place in minimally invasive PCa treatment.

\section{FT}

Despite the high incidence of $\mathrm{PCa}$, it is only responsible for $11 \%$ of all cancer deaths. ${ }^{1}$ This can be attributed to PSA-era screening protocols that allow earlier diagnosis of $\mathrm{PCa}$ while it is still localized and treatable. However, many investigators question whether we should treat all cases and postulate that we are overtreating patients. ${ }^{15}$ Recent figures indicate that $48-100$ patients must be treated to prevent a single cancer death, ${ }^{16,17}$ and even the most recent surgical techniques discussed previously are associated with morbidity and periprocedural complications. A recent study of radical prostatectomy specimens revealed that $78 \%$ of patients had indolent disease and could have been managed less aggressively. ${ }^{18}$

AS protocols are recommended for patients with low-risk disease, in an attempt to reduce overtreatment. In practice, the 10-year disease-specific survival of men who select AS is between $97 \%$ and $100 \%,{ }^{19,20}$ but although small, there is always the risk of missing the curative window. Recent analyses reveal that men who defer treatment have higher incidences of biochemical progression following definitive treatment, ${ }^{21}$ with up to $50 \%$ treatment failure in a large cohort of patients. ${ }^{19}$ This could be a reason for poor acceptance and adherence to AS by patients and physicians. Less than $10 \%$ of candidates select AS even when classified as very low risk. ${ }^{22}$ One study found that more than half of patients following AS drop out to seek definitive treatment and most do have an oncologic basis for doing so. ${ }^{20}$

FT can be viewed as the middle ground between radical therapy and AS. The concept of FT is extensively applied in other diseases, for example lumpectomy for breast cancer or local ablation of cervical cancer. ${ }^{23,24}$ The goal of FT is to ablate the tumor, specifically leaving nondiseased tissue and the surrounding critical structures unaffected. In doing so, FT could avoid the side effects of radical therapy and provide the oncologic control that AS lacks. By nature, FT is minimally invasive and should offer fewer periprocedural complications compared with radical whole-gland treatments.

Although the number of patients that have been treated with FT is low, there are published results of an international panel stating which patients are prime candidates for $\mathrm{FT}^{25}$ Patients should have low- to moderate-risk disease and favorable tumor topography. Although unifocal or unilateral disease would be most effectively treated with FT, there is an increasing body of literature suggesting that even multifocal bilateral disease might be effectively controlled with FT. This arises from the "index lesion" hypothesis. This theory hypothesizes that progression and metastasis arise from a single aggressive focus, the index lesion. ${ }^{26}$ Additional lowvolume satellite foci are more likely to harbor less aggressive disease. ${ }^{27}$ It follows that identifying and treating the index lesion will decrease risk of progression and cancer death.

\section{Practical considerations of FT Accuracy of PCa staging}

The indications for FT rely on accurate staging of PCa, but this is not always feasible. Studies of radical prostatectomy 
specimens of patients initially considered to have clinically insignificant disease reveal that $25 \%$ of patients are reclassified and $8.5 \%$ have extra-capsular extension. ${ }^{28}$ Understaging could have serious implications for patients managed conservatively with AS or FT, and this should be factored into treatment decisions.

Some of the inaccuracy of staging is due to biopsy sampling error associated with sextant biopsy. In some cases, extensive biopsy schemes have been shown to improve staging accuracy, but this is still a matter of debate. ${ }^{29} \mathrm{~A}$ study of men initially diagnosed with insignificant disease underwent 32 -core saturation biopsy, and $70 \%$ were reclassified. ${ }^{30}$ Conversely, another study found no increase in prognostic information for tumor volume or extra-capsular extension in patients with $>6$ cores. ${ }^{31} \mathrm{~A}$ recent comparison of 24-core perineal and transrectal biopsy schemes revealed no significant differences in PCa detection. ${ }^{32}$

Diagnostic imaging may play a role in identifying patients for FT. ${ }^{33}$ Excellent soft-tissue contrast is attained with magnetic resonance (MR), but standard T2 weighted images miss the majority of foci $<5 \mathrm{~mm}$ and around $10 \%$ of larger (>10 mm) foci. ${ }^{34}$ More sophisticated techniques such as dynamic contrast enhanced MR, diffusion weighted imaging, and MR spectroscopic imaging offer better characterization of PCa focality, stage, and size. ${ }^{35-37}$ Some FT protocols have integrated these multiparametric MR techniques into pretreatment staging in order to target and contour the lesion suspected of being the index lesion for accurate treatment targeting. ${ }^{38,39}$

Another recently developed technique that combines the advantages of imaging and biopsy is stereotactic biopsy. Early trials of multiparametric MR transrectal ultrasound (MR-TRUS) fusion systems report $44 \%$ improved sampling of significant lesions over 12-core random biopsy alone. ${ }^{40}$ Further, the targeted biopsy schema allow upstaging of lesions that may have been classified as insignificant by 12-core biopsy. ${ }^{41}$ MR-TRUS fusion systems with 3D/2.5D registration have reported $2.4 \mathrm{~mm}$ precision in phantoms and motion compensation using a closed-loop control system in humans. ${ }^{42}$ Real-time 3D ultrasound and multiparametric MR fusion have been reported in context of an FT trial. Stereotactic biopsy was used to select 14 patients for organ conservative high-intensity focused ultrasound (HIFU). ${ }^{43}$ Stereotactic biopsies might also play an important role in the future in enabling targeted FT in patients where imaging is negative for cancer. Lastly, navigated stereotactic biopsy might play a role in validating complete ablation post procedure. One study reported feasibility of documenting biopsy location with image fusion ${ }^{44}$ and has shown the possibility of achieving consistency with re-biopsy sampling.

\section{Evaluation of treatment efficacy}

Efficacy of most contemporary treatments is evaluated by the previously described trifecta. This poses a challenge to FT evaluation as there is currently no validated PSA threshold that would be considered fulfillment of the oncological aspect of the trifecta. After lesion-targeted FT and even hemiablation, most of the gland remains vital, ${ }^{45,46}$ hence the PSA does not reach undetectable levels. New standards of treatment efficacy would have to be implemented. Follow-up with PSA kinetics might prove to be one solution, and success could be considered a stable PSA with long doubling time ( $>3$ years) ${ }^{46}$ Another option is using imaging as a surrogate endpoint with or without repeat biopsies. ${ }^{46}$

\section{FT strategies and results}

Energy modalities currently being investigated for FT include cryotherapy, HIFU, focal laser ablation (FLA), photodynamic therapy (PDT), and irreversible electroporation (IRE). Cryotherapy uses extreme low temperature (typically $-35^{\circ} \mathrm{C}$ to $\left.-40^{\circ} \mathrm{C}\right)^{47}$ to cause freeze-thaw injury to target tissue. This technique requires percutaneous insertion of argon or nitrogen probes. HIFU is a noninvasive transrectal or transurethral application of high-intensity acoustic waves that damage target through hyperthermia and cavitation. FLA requires percutaneous insertion of a laser fiber and causes local photothermal destruction of the tissue. PDT causes reactive oxygen cytotoxicity when photosensitizing agents are excited by the output of a percutaneous laser fiber. IRE involves placing electrodes into the target lesion and delivering successive microsecond pulses of direct current. The process creates nanopores in the plasma membrane, inducing apoptotic cell death.

The outcome of these therapies tends to be related to the extent of the ablation and to accurate real-time monitoring of the ablation. The thermal ablative methods (HIFU, FLA, and cryotherapy) allow for close temperature monitoring to ensure that therapeutic temperatures are reached only in the target volume.

Organ-conservative cryotherapy has been reported with adequate oncologic control. ${ }^{47-49}$ Unilateral nerve sparing has been attempted, and data are now available for 48 patients followed for a mean of 4.5 years. Stable PSA was reported in $94 \%$ of patients, and of the 24 patients biopsied regularly, there was no evidence of local recurrence in the treated area. Excellent continence (100\%) and preservation of preoperative 
sexual function was reported ( $90 \%$ of men who were potent before treatment remained potent) ${ }^{49} \mathrm{~A}$ similar study reported a $96 \%$ negative biopsy rate, $93 \%$ free of biochemical recurrence, and $88.9 \%$ potency preservation. ${ }^{48} \mathrm{~A}$ study of $60 \mathrm{FT}$ cases reported similar figures. ${ }^{47}$

An FLA trial fused 3D ultrasound with MR imaging to guide transperineal FLA in 12 patients, and temperature was monitored with fluoroptic probes. No perioperative complications occurred, and mild, self-limiting side effects included fatigue, perineal discomfort, hematuria, and hematospermia. Investigators reported no significant decline in sexual or urinary function. Post-treatment biopsy indicated $67 \%$ of patients were free of disease in the target area. ${ }^{39}$ Histopathologic analysis of radical prostatectomy specimens initially treated with FLA has validated treatment efficacy, revealing homogeneous coagulative necrosis of the target volume with no remaining viable cells. ${ }^{45}$ Later, the same group performed fully MR-guided and monitored FLA in two patients without complication. Two weeks post-treatment contrast-enhanced MR imaging revealed devascularization of the target with no evidence of residual disease, damage to the rectum, or damage to the neurovascular bundles. ${ }^{38}$

The majority of reports of focal HIFU have been hemiablative. In a long follow-up of hemiablation in 12 patients, five patients $(41 \%)$ required subsequent treatment for recurrence or treatment failure, but not for 5-10 years after first HIFU. The authors report urinary tract infections in two patients and acute urinary retention in one, but a stabilization of international prostate symptom scores at 1 year and overall cancer-specific survival of $100 \%{ }^{50}$ Another group reported early cancer control with a cohort of 20 patients treated with hemiablation. The trifecta of continence, erections, and cancer control was achieved in $89 \%$ of patients. ${ }^{46}$ A comparison of whole to hemi-gland therapy revealed no significant differences in biochemical disease free survival, but no differences in quality of life measures were noted either. ${ }^{51}$ Conversely, Beerlage et al report that patients with selective lesion-targeted HIFU have reduced local control based on biopsy findings and increased PSA nadir. ${ }^{52}$

There is one report in the literature regarding focal PDT. Six men were treated on this protocol. After injecting meso tetra hydroxyl phenyl chlorin, light was administered to the lobe with biopsy-proven PCa. Adverse effects were observed, including irritative voiding symptoms in all patients for 2 weeks following treatment, one case of sepsis and one case of stress incontinence. In terms of cancer control, PSA decreased in most patients but biopsy results revealed at least one positive core in each patient following treatment, but it is unclear as to where the tissue was obtained. ${ }^{53}$ Several trials using the PDT compound WST11 are underway in an attempt to better define the role of focal PDT in PCa patients.

IRE is a relative new ablative technology. Most investigations into IRE have been in-vitro tests on the $\mathrm{PC} 3$ cell line, ${ }^{54}$ and more recently IRE for prostate has been evaluated in a canine model. ${ }^{55} \mathrm{~A}$ cohort of 16 men with PCa underwent focal IRE, and no periprocedure complications were reported. Post-treatment biopsy showed no viable $\mathrm{PCa}$ remaining in the targeted region..$^{56}$ The most promising aspect of this nonthermal modality is that the IRE should not theoretically damage nerves or blood vessels while completely ablating the tumor parenchyma. Experimental models of the prostate revealed that the electric field inside vessels and axons was nil compared with the high field distribution in surrounding tissue ${ }^{57}$ Also, IRE does not damage extracellular matrix proteins, so the interstitial architecture remains intact. The possible impact of IRE on nerves is still a matter of debate. Electrophysiologic studies of a sciatic nerve contained within an electroporated zone reveal that they remain excitable and conductive both immediately after IRE and 2 weeks later. ${ }^{58}$ In the human trial, potency was conserved. ${ }^{56}$ A different group performed IRE on a porcine sciatic nerve to evaluate the effect of direct IRE on nerves ${ }^{59}$ and reported signs of injury including axonal swelling, fragmentation, and loss, in addition to coagulative necrosis and immune infiltrate, but the electrophysiology was not assessed.

Thus far, neither therapy has emerged as most effective or free from morbidity. The recent interest in FT can be seen in the literature, but there are few reports of treatment efficacy. ${ }^{46,60-63}$ Each energy modality must be investigated with large cohorts and longer follow-up to determine their role in treating $\mathrm{PCa}$ and within the FT paradigm.

\section{Conclusion}

$\mathrm{PCa}$ is being increasingly diagnosed in younger and healthier men. These men are in the prime of their lives and desire an effective treatment that will leave them cancer free, reduce the impact on their quality of life, and allow them to go back to their normal activities shortly after the procedure. In this setting, new technologies have been developed to decrease the morbidity of traditional radical procedures. Robotic prostatectomy is an established procedure, while FT is a promising novelty that might shortly join as part of the armamentarium to treat patients with $\mathrm{PCa}$.

\section{Disclosure}

The authors have no conflicts of interest regarding this publication. 


\section{References}

1. Jemal A, Siegel R, Xu J, Ward E. Cancer statistics. 2010. CA Cancer J Clin. 2010;60(5):277-300.

2. Ferlay J, Autier P, Boniol M, Heanue M, Colombet M, Boyle P. Estimates of the cancer incidence and mortality in Europe in 2006. Ann Oncol. 2007;18(3):581-592.

3. Gallina A, Chun FK, Suardi N, et al. Comparison of stage migration patterns between Europe and the USA: an analysis of 11350 men treated with radical prostatectomy for prostate cancer. BJU Int. 2008;101(12): 1513-1518.

4. Desireddi NV, Roehl KA, Loeb S, et al. Improved stage and gradespecific progression-free survival rates after radical prostatectomy in the PSA era. Urology. 2007;70(5):950-955.

5. Walsh PC, Donker PJ. Impotence following radical prostatectomy: insight into etiology and prevention. J Urol. 1982;128(3):492-497.

6. Binder J, Kramer W. Robotically-assisted laparoscopic radical prostatectomy. BJU Int. 2001;87(4):408-410.

7. Ficarra V, Novara G, Artibani W, et al. Retropubic, laparoscopic, and robot-assisted radical prostatectomy: a systematic review and cumulative analysis of comparative studies. Eur Urol. 2009;55(5): 1037-1063.

8. Hu JC, Gu X, Lipsitz SR, et al. Comparative effectiveness of minimally invasive vs open radical prostatectomy. JAMA. 2009;302(14): 1557-1564.

9. Ficarra V, Novara G, Fracalanza S, et al. A prospective, non-randomized trial comparing robot-assisted laparoscopic and retropubic radical prostatectomy in one European institution. BJU Int. 2009;104(4): 534-539.

10. Di Pierro GB, Baumeister P, Stucki P, Beatrice J, Danuser H, Mattei A. A prospective trial comparing consecutive series of open retropubic and robot-assisted laparoscopic radical prostatectomy in a centre with a limited caseload. Eur Urol. 2011;59(1):1-6.

11. Bianco FJ Jr, Scardino PT, Eastham JA. Radical prostatectomy: long-term cancer control and recovery of sexual and urinary function (“trifecta"). Urology. 2005;66(5 Suppl):83-94.

12. Eastham JA, Scardino PT, Kattan MW. Predicting an optimal outcome after radical prostatectomy: the trifecta nomogram. J Urol. 2008;179(6):2207-2210; discussion 2210-2201.

13. Patel VR, Coelho RF, Chauhan S, et al. Continence, potency and oncological outcomes after robotic-assisted radical prostatectomy: early trifecta results of a high-volume surgeon. BJU Int. 2010;106(5):696-702.

14. Patel VR, Sivaraman A, Coelho RF, et al. Pentafecta: a new concept for reporting outcomes of robot-assisted laparoscopic radical prostatectomy. Eur Urol. 2011;59(5):702-707.

15. Etzioni R, Penson DF, Legler JM, et al. Overdiagnosis due to prostatespecific antigen screening: lessons from U.S. prostate cancer incidence trends. J Natl Cancer Inst. 2002;94(13):981-990.

16. Bul M, Schroder FH. Screening for prostate cancer - the controversy continues, but can it be resolved? Acta Oncol. 2011;50 Suppl 1:4-11.

17. Klotz LH, Nam RK. Active surveillance with selective delayed intervention for favorable risk prostate cancer: clinical experience and a 'number needed to treat' analysis. Can J Urol. 2006;13 Suppl 1:48-55.

18. Katz B, Srougi M, Dall'oglio M, et al. Are we able to correctly identify prostate cancer patients who could be adequately treated by focal therapy? Urol Oncol. Epub 2011 Mar 30.

19. Klotz L, Zhang L, Lam A, Nam R, Mamedov A, Loblaw A. Clinical results of long-term follow-up of a large, active surveillance cohort with localized prostate cancer. J Clin Oncol. 2010;28(1):126-131.

20. van den Bergh RC, Roemeling S, Roobol MJ, et al. Outcomes of men with screen-detected prostate cancer eligible for active surveillance who were managed expectantly. Eur Urol. 2009;55(1):1-8.

21. O'Brien D, Loeb S, Carvalhal GF, et al. Delay of surgery in men with low risk prostate cancer. J Urol. 2011;185(6):2143-2147.

22. Barocas DA, Cowan JE, Smith JA Jr, Carroll PR. What percentage of patients with newly diagnosed carcinoma of the prostate are candidates for surveillance? An analysis of the CaPSURE database. J Urol. 2008;180(4):1330-1334; discussion 1334-1335.
23. Recht A, Solin LJ. Breast-conserving surgery and radiotherapy in early-stage breast cancer: the importance of local control. Semin Radiat Oncol. 2011;21(1):3-9.

24. Martin-Hirsch PP, Paraskevaidis E, Bryant A, Dickinson HO, Keep SL. Surgery for cervical intraepithelial neoplasia. Cochrane Database Syst Rev. 2010(6):CD001318.

25. de la Rosette J, Ahmed H, Barentsz J, et al. Focal therapy in prostate cancer-report from a consensus panel. J Endourol. 2010;24(5):775-780.

26. Ahmed HU. The index lesion and the origin of prostate cancer. $N$ Engl J Med. 2009;361(17):1704-1706.

27. Karavitakis M, Winkler M, Abel P, Livni N, Beckley I, Ahmed HU. Histological characteristics of the index lesion in whole-mount radical prostatectomy specimens: implications for focal therapy. Prostate Cancer Prostatic Dis. 2011;14(1):46-52.

28. Oliveira IS, Pontes-Junior J, Abe DK, et al. Undergrading and understaging in patients with clinically insignificant prostate cancer who underwent radical prostatectomy. Int Braz J Urol. 2010;36(3):292-299.

29. Ploussard G, Epstein JI, Montironi R, et al. The contemporary concept of significant versus insignificant prostate cancer. Eur Urol. 2011;60(2): 291-303.

30. Boccon-Gibod LM, de Longchamps NB, Toublanc M, Boccon-Gibod LA, Ravery V. Prostate saturation biopsy in the reevaluation of microfocal prostate cancer. J Urol. 2006;176(3):961-963; discussion 963-964.

31. Grossklaus DJ, Coffey CS, Shappell SB, Jack GS, Cookson MS. Prediction of tumour volume and pathological stage in radical prostatectomy specimens is not improved by taking more prostate needle-biopsy cores. BJU Int. 2001;88(7):722-726.

32. Abdollah F, Novara G, Briganti A, et al. Trans-rectal versus transperineal saturation rebiopsy of the prostate: is there a difference in cancer detection rate? Urology. 2011;77(4):921-925.

33. Sooriakumaran P, Grover S, Tewari A. The success of focal therapy hinges on the success of imaging platforms. BJU Int. 2011;107(9): 1344-1346.

34. Ikonen S, Karkkainen P, Kivisaari L, et al. Magnetic resonance imaging of clinically localized prostatic cancer. J Urol. 1998;159(3): 915-919.

35. Sciarra A, Barentsz J, Bjartell A, et al. Advances in magnetic resonance imaging: how they are changing the management of prostate cancer. Eur Urol. 2011;59(6):962-977.

36. Delongchamps NB, Beuvon F, Eiss D, et al. Multiparametric MRI is helpful to predict tumor focality, stage, and size in patients diagnosed with unilateral low-risk prostate cancer. Prostate Cancer Prostatic Dis. Epub 2011 Mar 22.

37. Walz J, Marcy M, Pianna JT, et al. Identification of the prostate cancer index lesion by real-time elastography: considerations for focal therapy of prostate cancer. World J Urol. Epub 2011 May 26.

38. Raz O, Haider MA, Davidson SR, et al. Real-time magnetic resonance imaging-guided focal laser therapy in patients with low-risk prostate cancer. Eur Urol. 2010;58(1):173-177.

39. Lindner U, Weersink RA, Haider MA, et al. Image guided photothermal focal therapy for localized prostate cancer: Phase I trial. J Urol. 2009; 182(4):1371-1377.

40. Rastinehad AR, Chokshi S, Benjamin CJ, et al. Prostate cancer detection rates using a MR/TRUS fusion system: in patients with a previous negative prostate biopsy prostate cancer detection rates using a MR/TRUS fusion system: in patients with a previous negative prostate biopsy. 4th International Symposium on Focal Therapy and Imaging in Prostate and Kidney Cancer; 2011 May 25-27; Noordwijk, Amsterdam, The Netherlands.

41. Cornud F. Pre-biopsy multiparametric MRI with TRUS-MRI image fusion in patients with no previous biopsy and 4-10 ng/mL PSA levels. 4th International Symposium on Focal Therapy and Imaging in Prostate and Kidney Cancer; 2011 May 25-27; Noordwijk, Amsterdam, The Netherlands.

42. Xu S, Kruecker J, Guion P, et al. Closed-loop control in fused MR-TRUS image-guided prostate biopsy. Med Image Comput Comput Assist Interv. 2007;10(Pt 1):128-135. 
43. Baco E, Rud E, Klotz D, Svindland A, Eggesbo HB. Selecting patients for hemi HIFU using Koelis ${ }^{\circledR}$ 3D ultrasound and MRI fusion guided biopsies: a pilot study. Poster 57 presented at the 4th International Symposium on Focal Therapy and Imaging in Prostate \& Kidney Cancer, May 25-27, 2011, Noordwijk, Amsterdam, The Netherlands.

44. Turkbey B, Xu S, Kruecker J, et al. Documenting the location of prostate biopsies with image fusion. BJU Int. 2011;107(1):53-57.

45. Lindner U, Lawrentschuk N, Weersink RA, et al. Focal laser ablation for prostate cancer followed by radical prostatectomy: validation of focal therapy and imaging accuracy. Eur Urol. 2010;57(6):1111-1114.

46. Ahmed HU, Freeman A, Kirkham A, et al. Focal therapy for localized prostate cancer: a Phase I/II trial. J Urol. 2011;185(4):1246-1254.

47. Ellis DS, Manny TB Jr, Rewcastle JC. Focal cryosurgery followed by penile rehabilitation as primary treatment for localized prostate cancer: initial results. Urology. 2007;70(6 Suppl):9-15.

48. Bahn DK, Silverman P, Lee F Sr, Badalament R, Bahn ED, Rewcastle JC. Focal prostate cryoablation: initial results show cancer control and potency preservation. J Endourol. 2006;20(9):688-692.

49. Onik G, Vaughan D, Lotenfoe R, Dineen M, Brady J. The "male lumpectomy": focal therapy for prostate cancer using cryoablation results in 48 patients with at least 2-year follow-up. Urol Oncol. 2008; 26(5):500-505.

50. El Fegoun AB, Barret E, Prapotnich D, et al. Focal therapy with highintensity focused ultrasound for prostate cancer in the elderly. A feasibility study with 10 years follow-up. Int Braz J Urol. 2011;37(2):213-222.

51. Muto S, Yoshii T, Saito K, Kamiyama Y, Ide H, Horie S. Focal therapy with high-intensity-focused ultrasound in the treatment of localized prostate cancer. Jpn J Clin Oncol. 2008;38(3):192-199.

52. Beerlage HP, Thuroff S, Debruyne FM, Chaussy C, de la Rosette JJ. Transrectal high-intensity focused ultrasound using the Ablatherm device in the treatment of localized prostate carcinoma. Urology. 1999; 54(2):273-277.
53. Moore CM, Nathan TR, Lees WR, et al. Photodynamic therapy using meso tetra hydroxy phenyl chlorin (mTHPC) in early prostate cancer. Lasers Surg Med. 2006;38(5):356-363.

54. Rubinsky J, Onik G, Mikus P, Rubinsky B. Optimal parameters for the destruction of prostate cancer using irreversible electroporation. J Urol. 2008;180(6):2668-2674.

55. Onik G, Mikus P, Rubinsky B. Irreversible electroporation: implications for prostate ablation. Technol Cancer Res Treat. 2007;6(4): 295-300.

56. Rubinsky B, editor. Irreversible Electroporation. Series in Biomedical Engineering. Springer; 2010.

57. Daniels C, Rubinsky B. Electrical field and temperature model of nonthermal irreversible electroporation in heterogeneous tissues. J Biomech Eng. 2009;131(7):071006.

58. Neal RE 2nd, Rossmeisl JH Jr, Garcia PA, Lanz OI, HenaoGuerrero N, Davalos RV. Successful treatment of a large soft tissue sarcoma with irreversible electroporation. J Clin Oncol. 2011;29(13): e372-e377.

59. Schoellnast H, Monette S, Ezell PC, et al. Acute and subacute effects of irreversible electroporation on nerves: experimental study in a pig model. Radiology. 2011;260(2):421-427.

60. Lindner U, Trachtenberg J, Lawrentschuk N. Focal therapy in prostate cancer: modalities, findings and future considerations. Nat Rev Urol. 2010;7(10):562-571.

61. Nguyen CT, Jones JS. Focal therapy in the management of localized prostate cancer. BJU Int. 2011;107(9):1362-1368.

62. Moore CM, Pendse D, Emberton M. Photodynamic therapy for prostate cancer - a review of current status and future promise. Nat Clin Pract Urol. 2009;6(1):18-30.

63. Turpen R, Rosser CJ. Focal therapy for prostate cancer: revolution or evolution? BMC Urol. 2009;9:2.
Open Access Surgery

\section{Publish your work in this journal}

Open Access Surgery is an international, peer-reviewed, open access journal that focuses on all aspects of surgical procedures and interventions. Patient care around the peri-operative period and patient outcomes post surgery are key topics. All grades of surgery from minor cosmetic interventions to major surgical procedures are covered. Novel techniques

\section{Dovepress}

and the utilization of new instruments and materials, including implants and prostheses that optimize outcomes constitute major areas of interest. The manuscript management system is completely online and includes a very quick and fair peer-review system. Visit http://www.dovepress.com/ testimonials.php to read real quotes from published authors. 